\title{
Characterization of interphase conditions in composite materials
}

\author{
P. F. M. Meurs*, P. J. G. Schreurs, T. Peijs and H. E. H. Meijer \\ Eindhoven University of Technology, Centre for Polymers and Composites, PO Box 513, \\ 5600 MB Eindhoven, The Netherlands
}

\begin{abstract}
A mixed experimental-numerical method is presented which can be used to characterize interphases in composite materials. This method is marked by using measured field quantities, such as displacements and strains, and finite element simulations of the experiments to fit interphase parameters using an iterative minimum variance estimation algorithm. Field quantities are measured using marker fields in the vicinity of the interphase. The markers are applicd using the clectron beam of a scanning electron microscope (SEM). A marker grid, consisting of dots with a diameter of $0.2 \mu \mathrm{m}$, is placed on the surface of unidirectional glass fibre-reinforced epoxies. The displacement field is determined by calculating the relative displacements of the markers during transverse tensile experiments in a SEM. Subsequently, the experiment is modelled using the finite element method. The positions of the border markers of the marker grid define the geometry of the model, and the displacements of these markers the boundary conditions. A mathematical interphase model is included. The parameters, quantifying the mechanical behaviour of the interphase, are fitted using the iterative parameter estimation procedure. Based on the presented results, it can be concluded that the method is a promising tool for the characterization of interphases.
\end{abstract}

(Keywords: interphase; mechanical properties; scanning electron microscopy; strain field measurements)

\section{INTRODUCTION}

Composite materials are known to have excellent stiffness and strength characteristics in the fibre direction, but in the transverse direction these properties are relatively poor. Mechanical loading in the transverse direction will lead to the initiation and propagation of widespread microstructural damage, often starting with interphasial failure, followed by fracture of the matrix and/or fibres. In the design process, the mechanical behaviour of composites has to be described on a macroscopic scale by a constitutive relation between stresses and strains. The mechanical properties of the components of the composite, i.e. the fibre, matrix and interphase or interphasial region, determine this relation. Of these, the properties of the interphase, such as the Young's modulus, tensile strength, shear strength and frictional behaviour, are particularly difficult to determine. Although a lot of work has been done on this subject-mainly in shear loading - using experiments such as pull-out, microdebond, fragmentation or microindentation tests ${ }^{1,2}$, a complete characterization has not yet been achieved. The problems in finding an accurate description of interphase properties are mainly due to the

* To whom correspondence should be addressed inhomogeneity of the material, i.e. the high stiffness ratio between fibres and matrix and the inhomogeneous fibre distribution in a composite structure. In characterizing interphase conditions, experiments on model composites are often accompanied by numerical analyses, qualitatively describing the results of the experiments. To describe the influence of the interphase on transverse properties, numerical micromechanical simulations are often used as an effective tool ${ }^{3}$. However, these simulations are usually based on a simplified geometry and can only describe the influence of interphase properties, such as modulus and thickness, on the global transverse properties, but cannot be used to determine quantitative information on interphase properties.

Particularly in materials with complex behaviour, such as composite materials, new and better ways to determine the mechanical properties are necessary to obtain reliable constitutive data. Often, such methods are marked by the use of field quantities in experimental situations, where homogeneous stress and strain fields cannot be obtained. Consequently, several techniques have been developed to determine strain fields on the micrometre scale. In fibre fragmentation tests, laser Raman spectroscopy is used to determine the strains in fibres ${ }^{4,5}$. The stereo imaging technique is used to detcrminc strain fields at crack tips ${ }^{6.7}$. Also strain 
fields, detcrmincd by measuring marker displacements, are used to identify the local failure behaviour of short fibre-rcinforced composites ${ }^{8}$. On a scale of $\mu \mathrm{m}$, dot maps created by an electron beam in a scanning electron microscope (SEM) can also be used to measure strain fields directly'.

This paper describes a mixed experimental-numerical technique that offers an alternative for the characterization of inhomogeneous complex materials. Displacements and strains are measured in the vicinity of the interphase, and numerical analysis of the experiment becomes an essential part of the method. Hence, this mixed experimental-numerical approach integrates experiment and numerical analysis as two complementary aspects of one characterization technique. In the next section, the method will be described in more detail. The technique used to measure field quantities is described in the third section, after which the experimental procedures and some preliminary results are presented. Finally, some conclusions are offered.

\section{THE MIXED EXPERIMENTAL-NUMERICAL METHOD}

The method is based on the combination of measurement of displacements and strains, finite element modelling, and parameter estimation. The field quantities are measured during transverse experiments on unidirectional glass fibre-reinforced epoxies. By using an isotropic fibre like glass and a matrix material of which the bulk properties are known, micromechanical modelling is easier but, more importantly, the attention can be focused on the interphasial region. Figure 1 shows a schematic representation of the method.

Measurement of field quantities in the vicinity of the interphase is an essential part of this study and will be discussed in the next section. Marker displacement fields measured during experiments in a SEM, and displacement fields calculated with a finite element method, can be linked so as to find material parameters during an iterative procedure. The difference between measured and computed displacements is used to adjust the

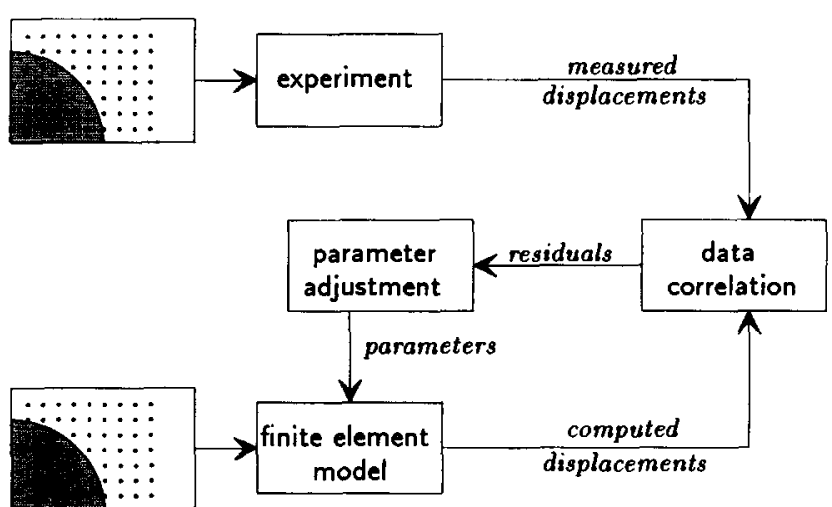

Figure 1 Schematic representation of the mixed experimentalnumerical method parameters describing the constitutive behaviour of the interphase. Parameters are estimated using an iterative minimum variance estimation algorithm.

\section{Finite element modelling}

The finite element model of the experiment requires a mathematical model and material parameters of the interphase. Generally, an interphase can be considered as a two-dimensional border between fibre and matrix, denoted as an interface, and interphases can be modelled as three-dimensional regions. If modelling the interphase as an interface, a spring model can be chosen. The mechanical properties are characterized by spring constants that relate normal and shear tractions to the normal and tangential displacements. Interphase layer models consider the interphase to be a region of specified thickness, having mechanical properties different from those of the fibre and matrix ${ }^{10,11}$. The interphase can then be modelled as isotropic, anisotropic, having constant or, for example, non-linear varying properties over its thickness. In this study, an interphase layer model is assumed to describe the mechanical properties of the interphase between a glass fibre and the bulk epoxy matrix.

In addition to the constitutive properties of the material under consideration, information about geometrical and loading data is needed. Numerical simulations of transverse tests are usually performed using plane strain conditions, and based on a simplified geometry consisting of a repeating unit with the fibres in either a square or a hexagonal array. This geometry is considered to be a representative volume element of the composite. Measuring field quantities by placing a marker grid on the specimen, however, offers the advantage that the geometry of the model is well defined (Figure 2), and geometrical assumptions are not needed. Because the displacements are measured at the surface of a specimen, the stress-strain state in the neighbourhood of a fibre has to be considered carefully. Therefore a three-dimensional numerical model has been analysed, which revealed that the out-of-plane stress is zero at the

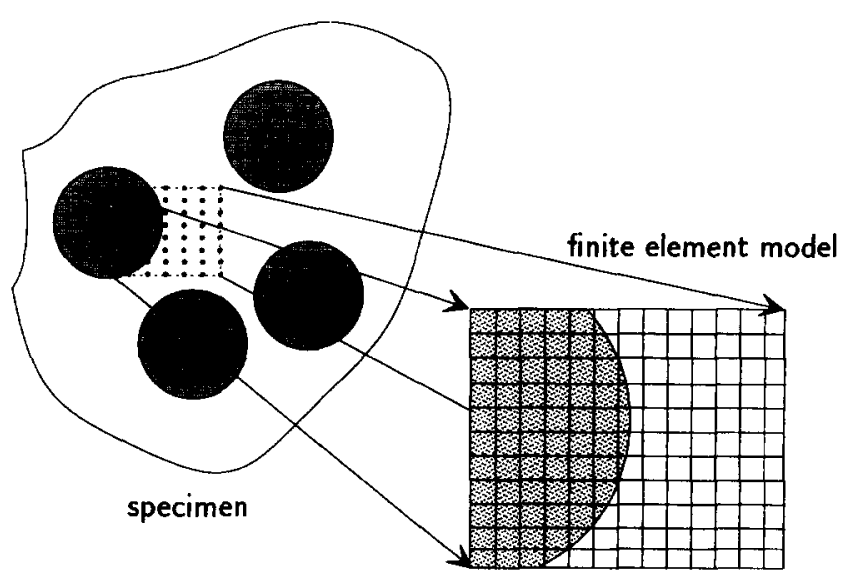

Figure 2 Local modelling of a part of the specimen 
surface and builds up to a constant value towards the inner part of the specimen. The out-of-plane strain was found to be zero over the thickness of the specimen. Based on these numerical results, it can be concluded that the experiment can be modelled two-dimensionally, using plane strain conditions.

The geometrical and loading data of the model are retrieved from the experiments. This local approach uses the positions of the border markers to define the geometry of the model (Figure 2). The displacements of these markers can be used as kinematic boundary conditions. This implies that forces cannot be part of the boundary conditions and thus only ratios between the different stiffness parameters can be determined. However, since the values of the parameters of the fibre and matrix are known, absolute values of interphase parameters can be fitted.

\section{Estimation of interphase parameters}

The estimation of interphase parameters comprises the comparison between the experimentally obtained displacement data of the inner markers of the grid (Figure 2) and the results of the finite element analysis $^{12,13}$. This comparison reveals updated estimations of the material parameters in the interphase model. To estimate these parameters, a sufficiently large series of measurement data is needed. These data are stored in a set of columns $\mathbf{y}_{k}, k=1, \ldots, N$, where index $k$ indicates a load step of an experiment. Each column $\mathbf{y}_{k}=\left[y_{1}, \ldots, y_{m}\right]_{k}^{T}$ contains the measured displacement data of material points. The unknown interphase parameters $x_{i}, i=1, \ldots, n$, quantifying the interphase behaviour, are assembled in a column $\mathbf{x}$. This column may contain interphase parameters such as Young's moduli, Poisson ratios or a non-linear function of matcrial constants. A finite element algorithm, represented by a function $\mathbf{h}_{k}(\mathbf{x})$, can calculate $\mathbf{y}_{k}$ when $\mathbf{x}$ is known. Function $\mathbf{h}_{k}(\mathbf{x})$ describes the dependency of observation $\mathbf{y}_{k}$ on $\mathbf{x}$ when no observation errors would be made. When including observation errors $\mathbf{v}_{k}$, the dependence can be written as:

$$
\mathbf{y}_{k}=\mathbf{h}_{k}(\mathbf{x})+\mathbf{v}_{k}
$$

The aim of parameter estimation is to construct a procedure or estimator which can calculate the unknown material parameters $\mathbf{x}$ for given values of the measured displacements. This procedure also requires knowledge of the statistics of the observation errors and the material parameters. The estimator minimizes the following sum of squares with respect to $\mathbf{x}$ :

$$
\begin{aligned}
S_{k}= & \left(\mathbf{y}_{k+1}-\mathbf{h}_{k+1}(\mathbf{x})\right)^{T} \mathbf{R}_{k+1}^{-1}\left(\mathbf{y}_{k+1}-\mathbf{h}_{k+1}(\mathbf{x})\right) \\
& +\left(\hat{\mathbf{x}}_{k}-\mathbf{x}\right)^{T} \mathbf{P}_{k}^{-1}\left(\hat{\mathbf{x}}_{k}-\mathbf{x}\right)
\end{aligned}
$$

where $\mathbf{R}_{k+1}$ and $\mathbf{P}_{k}$ are covariance matrices and $\hat{\mathbf{x}}_{k}$ is an estimate of $\mathbf{x}$ at load step $k$. The first term of $S_{k}$ represents the weighted sum of the displacement residuals, and the second term represents the weighted sum of the difference between the estimated and the true value of the interphase parameters. In a minimum variance estimator, $\mathbf{R}_{k+1}$ and $\mathbf{P}_{k}$ are the covariance matrices of the observation error and the estimation error:

$$
\begin{aligned}
E\left\{\mathbf{v}_{k+1}\right\} & =\mathbf{0} ; E\left\{\mathbf{v}_{k+1} \mathbf{v}_{k+1}^{T}\right\}=\mathbf{R}_{k+1} \\
E\left\{\hat{\mathbf{x}}_{k}-E\left\{\hat{\mathbf{x}}_{k}\right\}\right\} & =\mathbf{0} ; E\left\{\left(\hat{\mathbf{x}}_{k}-E\left\{\hat{\mathbf{x}}_{k}\right\}\right)\left(\hat{\mathbf{x}}_{k}-E\left\{\hat{\mathbf{x}}_{k}\right\}\right)^{T}\right\}=\mathbf{P}_{k}
\end{aligned}
$$

in which $E\{\}$ represents the expected value. The weighting matrices $\mathbf{R}_{k}$ and $\mathbf{P}_{k}$ are chosen on the basis of engineering judgement.

The estimation problem, defined by equations (1) and (2), can be solved iteratively by applying the following scheme.

\section{Parameter update:}

$$
\hat{\mathbf{x}}_{k+1}=\hat{\mathbf{x}}_{k}+\mathbf{K}_{k+1}\left(\mathbf{y}_{k+1}-\mathbf{h}_{k+1}\left(\hat{\mathbf{x}}_{k}\right)\right)
$$

with

$$
\mathbf{K}_{k+1}=\mathbf{P}_{k} \mathbf{H}_{k+1}^{T}\left(\mathbf{H}_{k+1} \mathbf{P}_{k} \mathbf{H}_{k+1}^{T}+\mathbf{R}_{k+1}\right)^{-1}
$$

Estimation error covariance update:

$$
\mathbf{P}_{k+1}=\left(\mathbf{I}-\mathbf{K}_{k+1} \mathbf{H}_{k+1}\right) \mathbf{P}_{k}
$$

with initial conditions:

$$
\left\{\begin{array}{l}
\hat{\mathbf{x}}=\overline{\mathbf{x}}_{0} \\
\mathbf{P}_{0}=\overline{\mathbf{P}}_{0}
\end{array}\right.
$$

where $\mathbf{I}$ is the unit matrix and $\overline{\mathbf{x}}_{0}$ and $\overline{\mathbf{P}}_{0}$ are initial conditions, values of which may be based on previous experiments.

For the calculation of $\mathbf{K}_{k+1}$ and $\mathbf{P}_{k+1}$, the non-linear function $\mathbf{h}_{k+1}$ is linearized with respect to the most recent estimation $\hat{\mathbf{x}}_{k}$, resulting in $\mathbf{H}_{k+1}$ :

$$
\mathbf{H}_{k+1}=\left(\frac{\partial \mathbf{h}_{k}(\mathbf{x})}{\partial \mathbf{x}}\right)_{\mathbf{x}=\dot{\mathbf{x}}_{k}}
$$

This iteration scheme is implemented in the finite element package DIANA ${ }^{14}$.

Using the estimation procedure, interphase conditions can be determined when a mathematical interphase model is available. In this model, assumptions for the interphase thickness and the number of parameters describing the mechanical behaviour have to be made. Consequently, the estimation of the interphase conditions may deviate from the true value because of modelling errors. The assumptions on which the interphase model is based can be checked by examining the residuals (the difference between the measured and calculated displacements) after the last iteration step. Some structure in the residuals, which suggests that the residuals are not randomly distributed, may point to a model error. Based on such an evaluation, the interphase model may be adjusted.

\section{MEASUREMENT OF FIELD QUANTITIES}

This section describes the technique that is used to measure ficld quantities on a local scale, in the vicinity of 


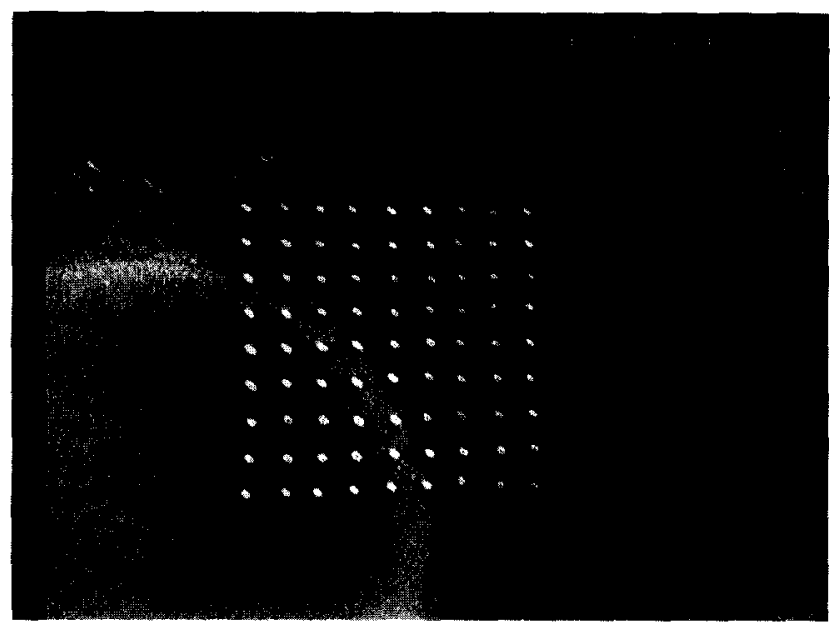

Figure 3 Example of a marker field on a glass/epoxy composite

an interphasial region. Displacements of material points during loading of a composite can be measured using displacentent markers on the surface of a specimen. Here, the markers are small raised dots, which can be created using a scanning electron microscope ${ }^{9}$. Before the markers can be obtained, specimens are polished and an electrically conductive gold-palladium coating is applied. The principle of creating raised dots is to use the contamination produced by the SEM. At very high magnifications, a SEM produces contamination on the surface of a specimen on top of the gold-palladium sputter coating. This contamination has a relatively high coefficient of thermal expansion. This can be exploited when the SEM is used at a very high magnification, with the resolution adjusted to give a small spot size. Using the SEM in 'spot mode' causes local heating of the contamination layer, followed by expansion, resulting in a raised dot. Repeating this procedure produces a marker field on the surface of a composite (Figure 3).

The raised dots appear as white dots with a diameter of approximately $0.2 \mu \mathrm{m}$. The relative displacements between these markers can be determined by comparing the deformed and the undeformed marker fields after the specimen is loaded using a SEM equipped with a loading stage. The relative marker displacements can subsequently be used to calculate the strain field.

\section{RESULTS}

Transverse tensile experiments were performed on continuous unidirectional glass fibre-reinforced epoxy composites. The advantage of using glass fibres is that they are isotropic, which means that the transverse properties are known. From the epoxy matrix, the bulk properties are also known. Composite plates were fabricated by wet-winding of fibres on a frame. After winding, the impregnated fibres were degassed in a vacuum oven to obtain a void-free composite. Plates were prepared by positioning the frame in an open-ended mould and placing the whole assembly in a hot-press.
The plate thickness and fibre volume fraction were controlled by metal stops of the required thickness, while during hot-pressing excess resin was squeezed out through the open ends of the mould. After curing at $120^{\circ} \mathrm{C}$ for $8 \mathrm{~h}$, the plate was post-cured for $12 \mathrm{~h}$ at $180^{\circ} \mathrm{C}$. This manufacturing procedure resulted in a flat plate of $160 \times 300 \times 1 \mathrm{~mm}$ with a fibre volume fraction of approximately $60 \%$. Using a diamond cutting wheel, the plates were cut into rectangular specimens, each with a length of $30 \mathrm{~mm}$ and a width of $10 \mathrm{~mm}$. Tensile specimens with curved edges (to minimize the region in which deformations will develop) were machined using a special grinding device. The curved edges were polished, using standard metallographic procedures, to a grit size of $1 \mu \mathrm{m}$. After polishing, a $100 \AA$ thick $60: 40$ goldpalladium sputter coating was applied for analysis in the SEM. A marker field was placed in the vicinity of an interphase, and the experiment was performed in a SEM equipped with a loading stage, at a displacement rate of $0.05 \mathrm{~mm} \mathrm{~min}^{-1}$.

Comparing the positions of the markers in unloaded and loaded configuration gives the displacement field shown in Figure 4. In this figure, the size of the displacements is enlarged by a factor of 2 . The displacements in a part of the glass fibre are approximately zero, which can be seen in the lower-left part of the displacement plot. In the upper-right part of this figure, representing a matrix-rich region, the largest displacements are measured.

The $x x$-component of the calculated strain field is shown in Figure 5. From the strain field, a jump in $\epsilon_{x x}$ can be seen near the interphasial region.

A finite element model of this experiment was made, the geometry of which is defined by the positions of the border markers in the reference configuration. The kinematic boundary conditions are given by the displacements of the border markers and the displacements of the inner markers are used to fit the model to the experiments. The interphase is assumed to be isotropic and have a thickness of $1 \mu \mathrm{m}$. The Young's moduli of the

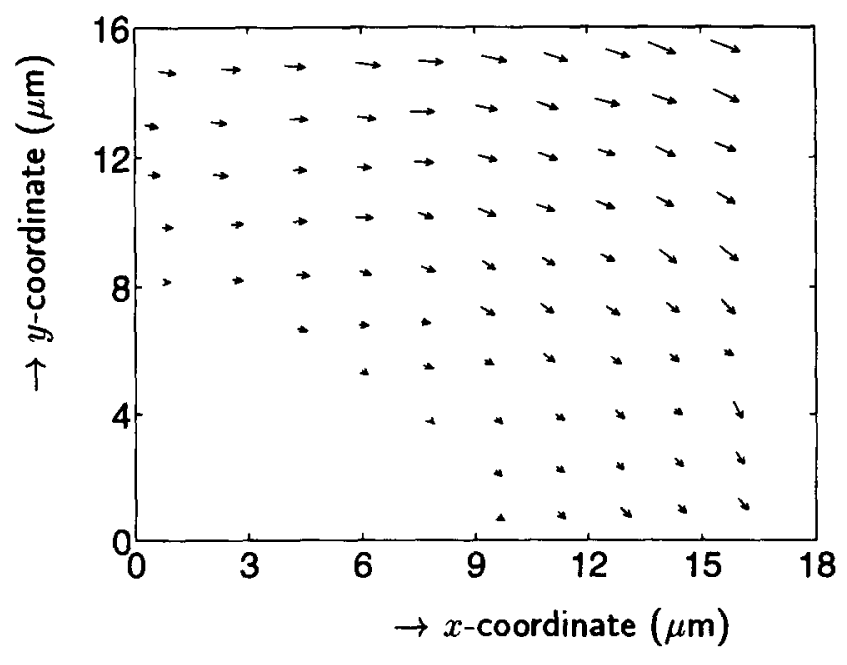

Figure 4 Measured displacements $(\times 2)$ near an interphase 


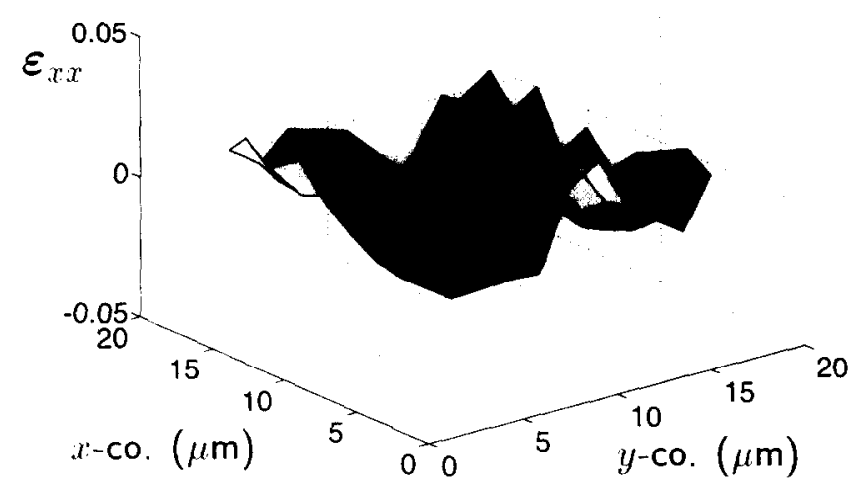

Figure 5 Calculated strain field near an interphase

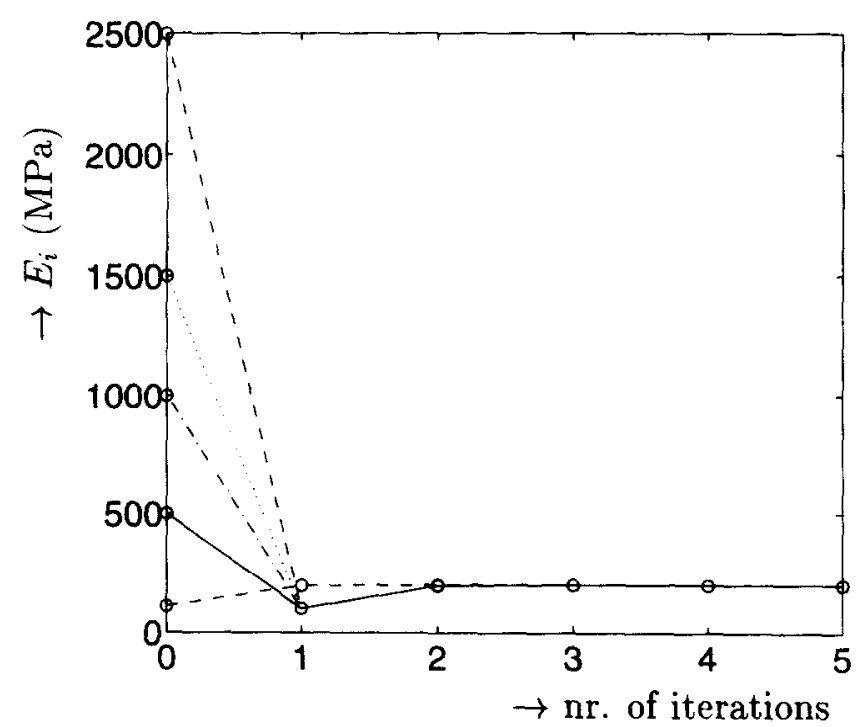

Figure 6 Estimation results for different initial estimations

fibre and matrix are $E_{\mathrm{f}}=70 \mathrm{GPa}$ and $E_{\mathrm{m}}=2.52 \mathrm{GPa}$, respectively. The Young's modulus of the interphase is fitted for five different initial estimates of $E_{\mathrm{i}}$. The results are given in Figure 6.

In all cases, the estimation algorithm converges to a constant value within three iterations. The estimated value of $E_{\mathrm{i}}$ is $200 \mathrm{MPa}$, approximately $10 \%$ of the matrix value. Although the authors do question the rather low value of the interphase modulus, these preliminary experiments show that the identification of material parameters itself is successful. The presented method for interphase characterization offers an alternative to existing methods, especially in the transverse loading direction. Therefore, currently the whole trajectory of experimenting, measuring marker displacements and modelling is being evaluated and optimized. In future experiments and estimations, validation of the interphase model by checking the displacement residuals after the final iteration will lead to a better description.

\section{CONCLUSIONS}

In this paper, a novel method for the characterization of interphase conditions in composite materials has been presented. The key aspect of this method is the combination of displacement field measurements during transverse loading with finite element modelling and parameter estimation. Displacements are measured on the micrometre scale in the vicinity of an interphase using raised dots on the surface of a specimen. These dots are created using a SEM. In situ tensile experiments in the SEM revealed a displacement field. The marker field defines the geometry of the finite element model, which is advantageous because geometrical assumptions about the fibre packing array are not needed. The displacements of the border markers are used as kinematic boundary conditons. Material parameter estimation is performed using a minimum variance estimation algorithm. Although the numerical simulations show that parameter estimation is possible, the accuracy of the displacement measurements can still be improved. Information on modelling errors, for example, based on a study of displacement residuals, can be used to provide tools for interphase model revision.

Based on the preliminary results presented in this paper, it can be concluded that the proposed experimental-numerical method is a promising tool for the characterization of interphase properties in composite materials.

\section{ACKNOWLEDGEMENTS}

This study was part of a research project financially supported by the Dutch Technology Foundation (STW). The authors wish to thank W. L. Bradley, C. R. Corleto and the staff of the Microscopy Center of Texas A\&M University (USA) for their assistance in developing the technique of obtaining displacement markers with a scanning electron microscope.

\section{REFERENCES}

1 'Interfacial Phenomena in Composite Materials '91', Proc. IPCM-2 (Eds I. Verpoest and F.R. Jones), ButterworthHeineman Ltd, Oxford, 1991

2 Proc. IPCM-3, Composites 1994, 25(7), special issue

3 de Kok, J.M.M. and Peijs, T., Deformation, yield and fracture of unidirectional composites in transverse loading: 2 . The influence of fibre-matrix adhesion. Composites in press

4 Galiotis, C., Young, R.J. and Batchelder, D.N., J. Polym. Sci. Polym. Phys. 1983, 21, 2483

5 Galiotis, C., A study of mechanisms of stress transfer in continuous- and discontinuous-fibre model composites by laser Raman spectroscopy. Compos. Sci. Technol. 1993, 48, 15

6 Davidson, D.L., Chan, K.S., McMinn, A. and Leverant, G.R., Micromechanics and fatigue crack growth in an alumina-fiberreinforecd magnesium alloy composite. Metall. Trans. A 1989 , 20A, 2369

7 Franke, E.A., Wenzel, D.J. and Davidson, D.L., Measurement of microdisplacements by machine vision photogrammetry (DISMAP). Rev. Sci. Instrum. 1991, 5, 1270

8 Geers, M.G.D., Peijs, T., de Borst, R and Brekelmans, W.A.M. Experimental dynamic analysis of damage evolution in shortfibre-reinforced composite materials. In 'Proc. ICCM-10', Whistler, BC, Canada, August 1995

9 Corleto, C.R., Bradley, W.L. and Brinson, H.F., An experimental micromechanics measurement technique for sub-micron domains. J. Mater. Sci, submitted 
10 Jayaraman, K., Reifsnider, K.L. and Swain, R.E., Elastic and thermal effects in the interphase: Part I. Comments on characterization methods. J. Compos. Technol. Res. 1993, 15(1), 3

11 Jayaraman, K., Reifsnider, K.L. and Swain, R.E., Elastic and thermal effects in the interphase: Part II. Comments on modeling studies. J. Compos. Technol. Res. 1993, 15(1), 14

12 Hendrix, M.A.N., Oomens, C.W.J., Jans, H.W.J., Janssen, J.D. and Kok, J.J., A numerical experimental approach for the characterization of composites. In 'Proc. 9th Int. Conf.
Exp. Mech.' (Ed. V. Askegaard), Aabt Tryk, Copenhagen, 1990 13 van Ratingen, M.R., Oomens, C.W.J. and Janssen, J.D., Mechanical testing with non uniform strain fields. In 'Composite Testing and Standardisation' (Eds P.J. Hogg, G.D. Sims, F.L. Matthews, A.R. Bunsell, A. Massiah), European Association for Composite Materials, Bordeaux, 1992

14 'DIANA Finite Element Analysis User Manuals', TNO for Building Materials Building Structures, Rijswijk, The Netherlands, 1992 